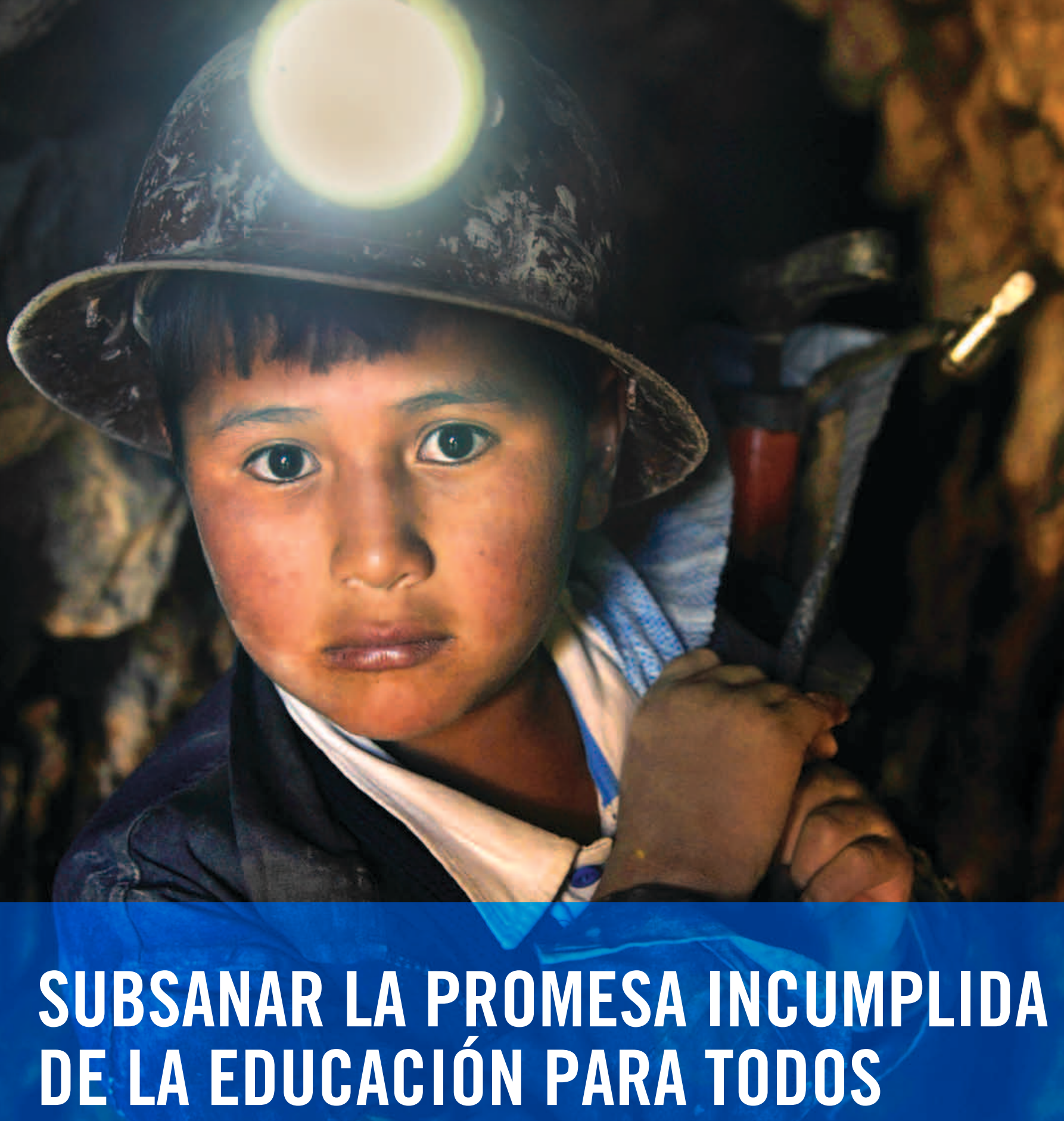

Conclusiones de la Iniciativa Global por los Niños Fuera de la Escuela

RESUMEN EJECUTIVO

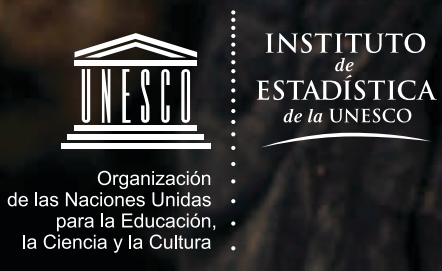

\title{
unicef
}




\section{SUBSANAR LA PROMESA INCUMPLIDA DE LA EDUCACIÓN PARA TODOS Conclusiones de la Iniciativa Global por los Niños Fuera de la Escuela}

RESUMEN EJECUTIVO 


\section{Instituto de Estadística de la UNESCO}

El Instituto de Estadística de la UNESCO es la oficina de estadística de la UNESCO y es depositario de la ONU de las estadísticas mundiales en los campos de la educación, la ciencia y la tecnología, la cultura y las comunicaciones. Es la fuente oficial de los datos utilizados para hacer seguimiento a los progresos relacionados con la Educación para Todos y las metas conexas de los Objetivos de Desarrollo del Milenio (ODM).

La base de datos de educación del Instituto de Estadística de la UNESCO es la más extensa del mundo y abarca un amplio conjunto de indicadores: desde la matriculación de niñas en la escuela primaria hasta la movilidad de los estudiantes universitarios. Se actualiza tres veces al año sobre la base de los resultados de la encuesta anual sobre educación que el Instituto realiza en más de 200 países y territorios. Establecido en 1999, el Instituto de Estadística de la UNESCO está al servicio de los Estados Miembros y del sistema de las Naciones Unidas, así como también de las organizaciones intergubernamentales y no gubernamentales, institutos de investigación, universidades y particulares interesados en obtener datos de alta calidad. Nuestro objetivo primordial es mejorar las posibilidades y las condiciones de vida de los niños y los adultos del mundo entero, mediante la producción de los datos que se requieren para el diseño y la aplicación de políticas e intervenciones eficaces.

\section{Fondo de las Naciones Unidas para la Infancia (UNICEF)}

UNICEF promueve los derechos y el bienestar de todos los niños y niñas en todo lo que hacemos. Junto a nuestros aliados, trabajamos en 190 países y territorios para transformar este compromiso en acciones prácticas, centrando especialmente nuestros esfuerzos en llegar a los niños más vulnerables y excluidos para el beneficio de todos los niños, en todo el mundo.

UNICEF es una parte vital de los esfuerzos internacionales destinados a alcanzar los ODM sobre educación primaria universal en 2015. Una de sus contribuciones es actuar como secretaría de la Iniciativa de las Naciones Unidas para la Educación de las Niñas (UNGEI).

Publicado en 2015 por:

\section{Instituto de Estadística de la UNESCO}

C.P. 6128, Succursale Centre-Ville

Montréal (Québec) H3C 3J7

Canadá

Tel: +15143436880

Email: uis.publications@unesco.org

www.uis.unesco.org

(C) UNESCO-UIS 2015

ISBN: 978-92-9189-165-8

Ref: UIS/2015/ED/SD/8

DOI: http://dx.doi.org/10.15220/978-92-9189-165-8-sp

Diseño: Em Dash Design / www.emdashdesign.ca

Créditos fotográficos: Portada: @ Organización Internacional del Trabajo (OIT)/Crozet M. Interior: Shutterstock (p. 4); @ Ollivier Girard/Center for International Forestry Research (CIFOR) (p. 6); @ OIT/Ferry Latief 2012 (p. 12); @ DVIDSHUB/flickr (p.15)

Esta publicación se encuentra disponible en libre acceso bajo la licencia Attribution-ShareAlike 3.0 IGO (CC-BY-SA 3.0 IGO) (http://creativecommons. org/licenses/by-sa/3.0/igo/). Al utilizar su contenido, los usuarios aceptan las condiciones de utilización del repositorio de acceso libre de la UNESCO (http://www.unesco.org/open-access/terms-use-ccbysa-en).

Las denominaciones empleadas en esta publicación y la forma en que aparecen presentados los datos no implican, de parte de la UNESCO, juicio alguno sobre la condición jurídica de países, territorios, ciudades o zonas, o de sus autoridades, ni sobre la delimitación de sus fronteras o límites.

Los autores se hacen responsables por las ideas y opiniones que aquí expresan, las cuales no reflejan necesariamente las de la UNESCO, ni comprometen a la Organización.

Descargar la información que se presenta en este informe

En este informe se utiliza DataLinks, que permite descargar archivos Excel con los datos de la mayoría de las cifras y las tablas estadísticas. En la versión electrónica del presente informe, haga clic en el enlace ubicado al lado del icono de DataLinks. En la copia impresa, introduzca el enlace tras teclear http://dx.doi.org en su navegador de Internet.

Este informe debe citarse como:

Instituto de Estadística de la UNESCO y UNICEF (2015). Subsanar la promesa incumplida de la Educación para Todos Conclusiones de la Iniciativa Mundial sobre los Niños Sin Escolarizar. Montréal: Instituto de Estadística de la UNESCO.

http://dx.doi.org/10.15220/978-92-9189-165-8-sp 


\section{Prefacio}

La educación representa las esperanzas, sueños y aspiraciones de los niños, las familias, las comunidades y las naciones de todo el mundo, el medio más seguro para salir de la pobreza y un camino fundamental para contar con ciudadanos más sanos y productivos, y sociedades más sólidas. Por eso, no debe sorprender que cuando se pide a la gente que enumere sus prioridades, la educación ocupe el primer lugar en todas las encuestas.

A todos los niveles - desde las familias más pobres en las aldeas más remotas hasta los dirigentes que están trazando los futuros objetivos de desarrollo del mundo - existe un consenso: la educación es importante. Este consenso se ha traducido en acciones concretas, y ha llevado a las aulas a millones de niños que antes no recibían una educación. En los 15 años que han pasado desde que se presentaran los Objetivos de Desarrollo del Milenio - que establecieron la meta de lograr que todos los niños terminaran un ciclo completo de enseñanza primaria para 2015 - los últimos datos muestran que el número de niños y niñas en edad de asistir a la educación primaria que se encontraba fuera de la escuela descendió en un $42 \%$, siendo este porcentaje para las niñas solamente del $47 \%$, pese al rápido crecimiento de la población.

¿Por qué hay entonces todavía 58 millones de niños de todo el mundo, entre los 6 y los 11 años aproximadamente, que se encuentran fuera de la escuela? Cada uno de estos niños constituye un recordatorio riguroso de la promesa incumplida de lograr la educación primaria universal en la fecha límite original de 2015.

Subsanar la Promesa Incumplida de la Educación para Todos, un informe producido por el Instituto de Estadística de la UNESCO y UNICEF, no podía ser más oportuno. En un momento en que el mundo renueva su compromiso para avanzar hacia que todos los niños tengan su derecho a la educación, el informe explora por qué los progresos mundiales se han estancado desde principios de la década de 2000, cuando millones de niños llenaron las aulas de todo el mundo, y ofrece los datos y el análisis necesarios para avanzar y llegar a cada uno de los niños y niñas excluidos de la educación.

Con su interesante combinación de datos y análisis, este informe proporciona una evaluación de por qué algunos niños nunca llegan a un aula escolar; por qué hay niños que empiezan a estudiar mucho más tarde que otros; y por qué hay algunos que tienen más probabilidades que otros de abandonar los estudios antes de completar su educación. Nos recuerda, si es que necesitamos que nos lo recuerden, la necesidad crítica de obtener buenos datos que sirvan de base a las políticas educativas destinadas a reducir los obstáculos que siguen interponiéndose entre los niños y su derecho fundamental a la educación.

Este informe describe algunas de estas políticas y estrategias. Incluye una mayor atención a la mejora de la calidad de la educación para aumentar la probabilidad de que los niños vayan a la escuela y permanezcan en ella si la educación que se ofrece en las aulas se ajusta a sus propósitos. Además, dado el número alarmantemente elevado de adolescentes fuera de la escuela - 63 millones en todo el mundo en 2012 - aboga 
por establecer una educación secundaria universal sobre la base de las lecciones aprendidas desde 2000 en la educación primaria universal, y a partir de ellas.

Finalmente, este informe nos muestra a los niños más allá de las cifras. El pequeño que todos los días empuja una carretilla en un bazar de Kirguistán para ayudar a la manutención de su familia. La niña yemení que se vio obligada a retirarse de la escuela para casarse en contra de su voluntad. El niño que sufrió humillaciones en su escuela, en Sri Lanka, por no llevar unos zapatos en buen estado, y que dejó de estudiar cuando lo quisieron pasar a un curso inferior. El niño en Namibia que, a causa de una deficiencia auditiva no diagnosticada, lucha afanosamente para sacar adelante sus estudios. El niño refugiado de Siria que no es admitido en ninguna escuela debido a que todas están desbordadas en su capacidad.

A medida que la comunidad internacional renueva y amplía sus compromisos como parte del programa de desarrollo post-2015, debemos centrarnos en estos niños y en los millones que luchan para lograr el derecho a una educación y para cumplir sus sueños de un futuro mejor. Trabajando juntos y promoviendo una mayor inversión, podemos y debemos desmantelar los obstáculos que se interponen en su camino, uno por uno, y, al hacerlo, contribuir a cumplir nuestra promesa de educación para todos.

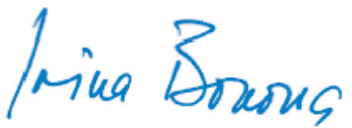

Irina Bokova

Directora General

UNESCO

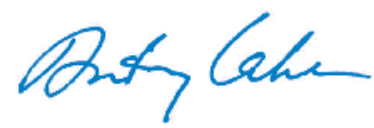

Anthony Lake

Director Ejecutivo

UNICEF

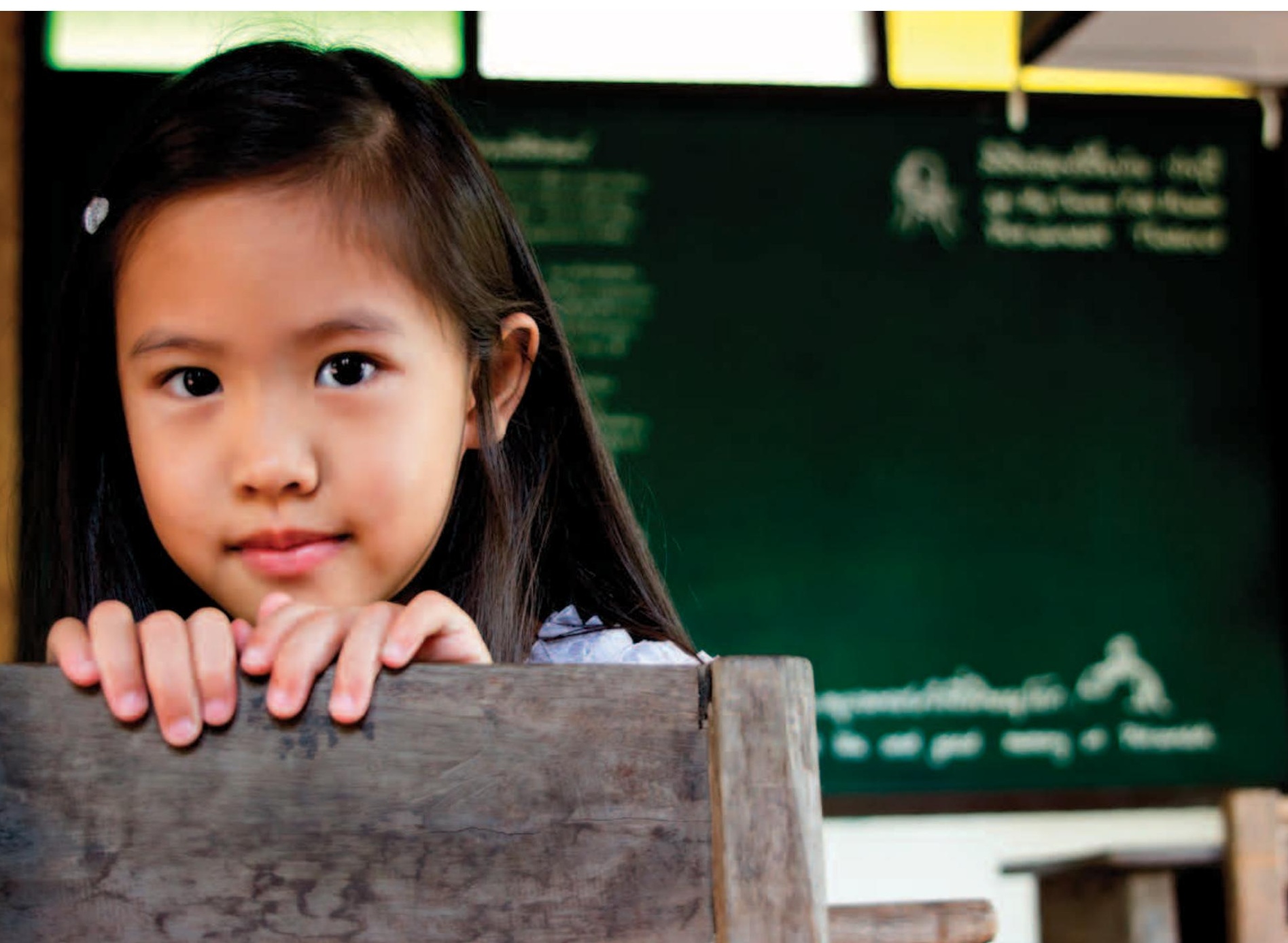




\section{Resumen ejecutivo}

\section{INTRODUCCIÓN}

Harish, un niño de 16 años de Polonnaruwa, contó que en varias ocasiones lo castigaron por asistir a la escuela sin los zapatos adecuados, pues sus padres carecían de los medios para comprarle unos nuevos. Como no le iba bien en sus estudios, el director quería bajarlo de curso. Sin embargo, con 14 años en ese momento, Harish prefirió abandonar la escuela a estudiar en un curso inferior.

Estudio sobre Sri Lanka de la Iniciativa Global por los Niños Fuera de la Escuela (UNICEF e Instituto de Estadística de la UNESCO, 2013a)

Las cifras se conocen, los datos se analizaron y las conclusiones son claras: a pesar de los grandes logros en matriculación escolar durante los últimos 15 años, el objetivo de la educación primaria universal no se cumplirá en 2015. La imposibilidad de alcanzar un objetivo que parecía realista y viable representa una promesa incumplida para millones de niños y niñas a los que se les continúa negando su derecho fundamental a la educación primaria.

Es verdad que se registraron considerables avances en la matriculación en el nivel primario, particularmente a comienzos de la década de 2000, impulsados en gran parte por los esfuerzos relacionados con los ODM y los objetivos de la Educación para Todos. Como resultado, el número de niños fuera de la escuela en edad de asistir a la primaria se redujo un 42\% entre 2000 y 2012 en todo el mundo. Pero el informe Fixing the Broken Promise of Education for All: Findings from the Global Initiative on Out-of-School Children (Subsanar la Promesa Incumplida de la Educación para Todos:
Los progresos en la tasa y números de niños fuera de la escuela se han estancado desde 2007

Un $\mathbf{9 \%}$ de niños en edad de cursar la educación primaria y un $17 \%$ de adolescentes con edad para estar en la secundaria están excluidos de la educación

Conclusiones de la Iniciativa Global por los Niños Fuera de la Escuela) confirma que millones de niños no se han beneficiado de esos progresos, que los avances no han sido equitativos y que se han estancado.

El informe Fixing the Broken Promise of Education for All se basa en estudios nacionales respaldados por los gobiernos de un diverso grupo de países que participan en la Iniciativa Global por los Niños fuera de la Escuela, puesta en marcha en 2010 y dirigida por UNICEF y el Instituto de Estadística de la UNESCO. Este informe reúne una amplia gama de fuentes de datos - cuantitativos y cualitativos - y revela información crucial sobre la escala y la magnitud de este problema, al tiempo que proporciona un análisis minucioso de los obstáculos para alcanzar la educación y las posibles soluciones de política. Participar en esta iniciativa significa para muchos países no solo una oportunidad sin igual para llamar la atención sobre los niños que permanecen invisibles y que carecen de voz, sino también para dar a conocer los rostros humanos que hay detrás de las cifras. 


\section{CONCLUSIONES PRINCIPALES}

El informe muestra que a pesar de los progresos conseguidos en lo que se refiere a matriculación en educación primaria, en todo el mundo hay 58 millones de niños y niñas en edad de asistir a ese nivel educativo (entre los 6 y los 11 años, aproximadamente) que no lo están haciendo (Instituto de Estadística de la UNESCO e Informe de Seguimiento de la Educación para Todos en el Mundo, 2014). De continuar las tendencias actuales, probablemente dos quintas partes de estos niños - es decir, 15 millones de niñas y 10 millones de niños - no conocerán jamás un aula escolar. En efecto, la mayoría de los 30 millones de niños y niñas que se encuentran fuera de la escuela en África Subsahariana nunca entrarán a una escuela.

Desde hace mucho tiempo se ha considerado que la educación primaria es esencial para el pleno desarrollo de los niños; sin embargo, cada vez se reconoce más que el primer ciclo de la enseñanza secundaria es decisivo para la adquisición de las habilidades necesarias para una vida sana y productiva, y para acceder a trabajos decentes. Existe un claro consenso en la comunidad internacional sobre la importancia de convertir la educación secundaria universal en un objetivo clave de la Agenda para el Desarrollo Post-2015. Pero en este punto el informe también señala que no se ha progresado lo suficiente, ya que 63 millones de adolescentes en edad de cursar el primer ciclo de secundaria están fuera de la escuela; esto es, 5 millones más que los niños en edad de cursar la primaria, aun cuando el número de estos últimos niños es el doble a nivel mundial.

Además, el progreso se ha estancado y prácticamente no se han registrado cambios en la tasa mundial ni en el número de niños que no asisten a la escuela desde 2007. Si bien el acceso a la enseñanza se elevó notoriamente a comienzos de la década de 2000, ha habido muy poco o ningún cambio en el número de niños sin matricular en todo el mundo desde 2007. La tasa global de niños en edad de primaria fuera de la escuela se ha estancado en cerca de un $9 \%$ durante los últimos siete años - más o menos 60 millones de niños, mientras que la tasa para los niños en edad de asistir al primer ciclo de la escuela secundaria sigue cercana a un 18\% (ver el Gráfico E1). En estos dos grupos de edad, las niñas siguen teniendo más probabilidades que los niños de no estar escolarizadas.

\section{La situación}

- 58 millones de niños en edad de cursar la primaria estaban fuera de la escuela en 2012. De ellos:

> Un 23\% asistió anteriormente a la escuela, pero la abandonó.

- Un 34\% es probable que ingrese a la escuela en el futuro.

> Un 43\% probablemente no ingresará nunca al sistema educativo.

- 63 millones de adolescentes en edad de cursar el primer ciclo de la educación secundaria se encontraban fuera de la escuela.

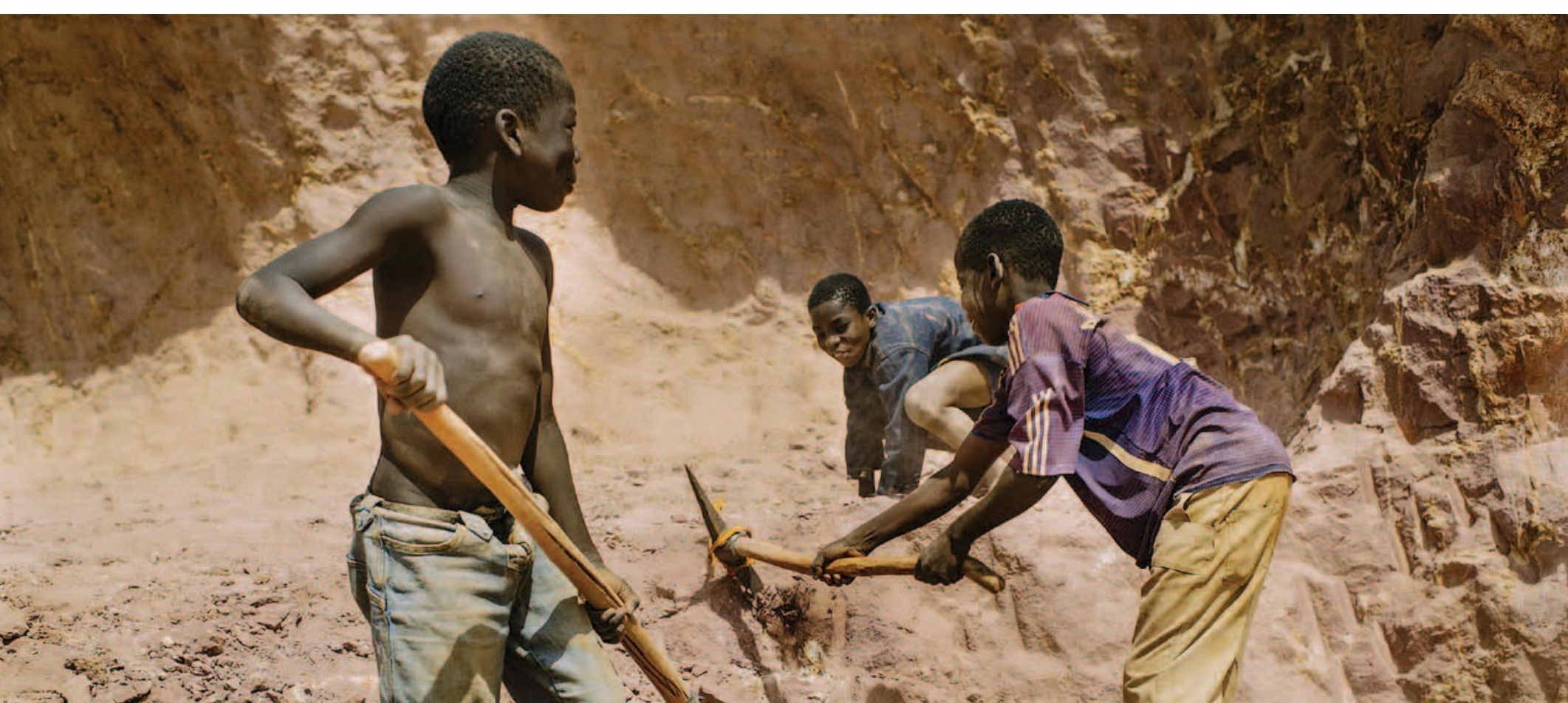


Un tercio de todos los niños en edad de cursar la primaria que están fuera de la escuela viven en África Occidental y Central, la región con la tasa más alta de niños no escolarizados. Aquí, más de uno de cada cuatro niños (un $31 \%$ de todas las niñas y un $23 \%$ de todos los niños varones) no va a la escuela; es decir, mucho más que en cualquier otra región. En África Oriental y Meridional y en Asia Meridional, otros 11 millones y 10 millones de niños, respectivamente, están fuera de la escuela. En términos relativos, la situación de Asia Meridional sin embargo era mucho mejor que la de África Subsahariana porque el 94\% de sus niños en edad de estudiar en la escuela primaria estaban matriculados, en comparación con el 85\% en África Oriental y Meridional y el 73\% en África Occidental y Central. Las tasas de niños fuera de la escuela eran menores en Asia Meridional y América Latina y el Caribe (6\%); en Europa Central y Oriental y la Comunidad de Estados Independientes (ECE/ CEI) y en Asia Oriental y el Pacífico (5\%); y en Europa Occidental, América del Norte y Australasia (4\%).

En el plano mundial, el número de adolescentes fuera de la escuela es similar al de los niños en esa condición, aun cuando en 2012 había casi el doble de niños en edad de asistir a la escuela primaria (650 millones) que de adolescentes en edad de cursar el primer ciclo de la educación secundaria (374 millones) (ver la Tabla E1). Es decir, pese a que el número de adolescentes es mucho menor, su probabilidad de estar fuera de la escuela es casi el doble que la de los niños en edad para la primaria (17\% y $9 \%$, respectivamente). Más del $40 \%$ de todos los adolescentes que no asisten a la escuela viven en Asia Meridional, y un tercio en África Subsahariana. Aun cuando en Asia Meridional se registraba un mayor número de adolescentes que no están escolarizados, África Occidental y Central tenía en 2012 la tasa más alta de adolescentes fuera de la escuela del primer ciclo de la secundaria, seguida de África Oriental y Meridional (27\%) y Asia Meridional (26\%). En Oriente Medio y África del Norte, un $12 \%$ de todos los adolescentes en edad de estudiar en el primer ciclo de la enseñanza secundaria no estaban matriculados. Al igual que en la primaria, los porcentajes más bajos de adolescentes que no están escolarizados se encontraron en Europa Occidental, América del Norte y Australasia (3\%), y en la región de ECE/CEI (5\%).

\section{Gráfico E1. Tasas promedios mundiales de niños fuera de la escuela con edades de asistir a la escuela primaria y al primer ciclo de la educación secundaria, 2000-2012}

$\%$

30

25

25

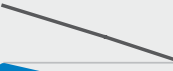

-

20

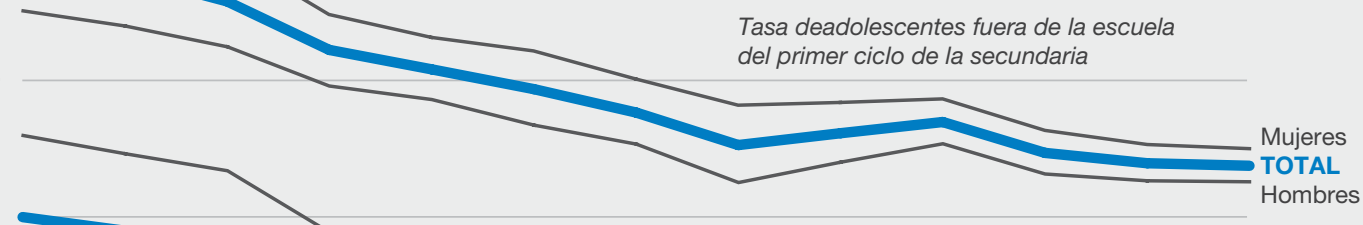

15

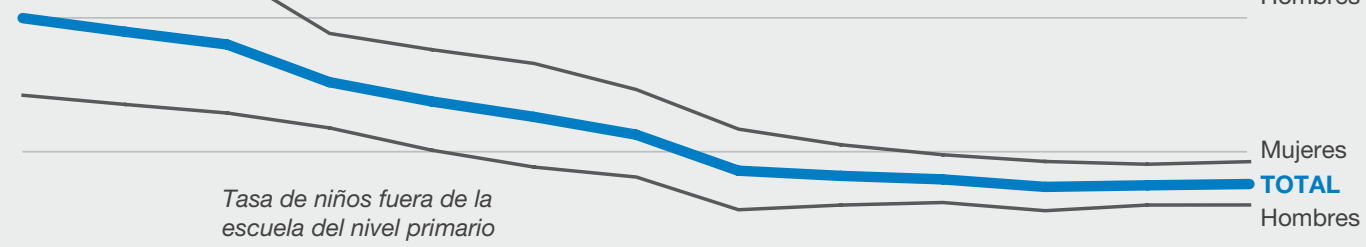

5

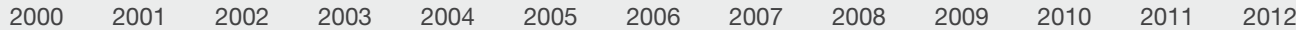

DataLink: http://dx.doi.org/10.15220/2014/ed/sd/7/f2.1 
Tabla E1. Niños en edad de primaria y adolescentes en edad de cursar el primer ciclo de la educación secundaria que se encuentran fuera de la escuela, 2012

\begin{tabular}{|c|c|c|c|c|c|c|c|c|c|c|c|c|}
\hline \multirow[b]{3}{*}{ Région } & \multicolumn{6}{|c|}{$\begin{array}{l}\text { Niños en edad de asistir a la escuela } \\
\text { primaria fuera de la escuela }\end{array}$} & \multicolumn{6}{|c|}{$\begin{array}{l}\text { Adolescentes en edad de asistir al primer } \\
\text { ciclo de la secundaria fuera de la escuela }\end{array}$} \\
\hline & \multicolumn{3}{|c|}{ Porcentaje } & \multicolumn{3}{|c|}{ Número (en millones) } & \multicolumn{3}{|c|}{ Porcentaje } & \multicolumn{3}{|c|}{ Número (en millones) } \\
\hline & HM & $\mathbf{H}$ & $\mathbf{M}$ & HM & $\mathbf{H}$ & $\mathbf{M}$ & HM & $\mathbf{H}$ & M & HM & $\mathbf{H}$ & M \\
\hline $\begin{array}{l}\text { EUROPA OCCIDENTAL, AMÉRICA } \\
\text { DEL NORTE, AUSTRALASIA }\end{array}$ & 3,5 & 3,6 & 3,4 & 2,2 & 1,2 & 1,1 & 2,7 & 2,6 & 2,8 & 1,0 & 0,5 & 0,5 \\
\hline AMÉRICA LATINA Y EL CARIBE & 6,3 & 6,5 & 6,1 & 3,8 & 2,0 & 1,8 & 7,5 & 7,7 & 7,3 & 2,8 & 1,5 & 1,3 \\
\hline ECE/CEI & 4,7 & 4,7 & 4,8 & 1,0 & 0,5 & 0,5 & 5,2 & 5,1 & 5,5 & 1,2 & 0,6 & 0,6 \\
\hline ASIA ORIENTAL Y EL PACÍFICO & 4,6 & 4,6 & 4,6 & 6,9 & 3,6 & 3,2 & 8,4 & 8,6 & 8,2 & 7,4 & 4,0 & 3,4 \\
\hline ASIA MERIDIONAL & 5,8 & 5,7 & 5,9 & 9,8 & 5,1 & 4,8 & 26,4 & 26,3 & 26,4 & 26,3 & 13,7 & 12,6 \\
\hline $\begin{array}{l}\text { ORIENTE MEDIO Y ÁFRICA DEL } \\
\text { NORTE }\end{array}$ & 9,3 & 7,6 & 11,1 & 4,3 & 1,8 & 2,5 & 11,7 & 9,4 & 14,1 & 2,9 & 1,2 & 1,7 \\
\hline ÁFRICA ORIENTAL Y MERIDIONAL & 15,1 & 13,6 & 16,6 & 11,0 & 5,0 & 6,0 & 26,9 & 24,2 & 29,5 & 8,5 & 3,8 & 4,6 \\
\hline ÁFRICA OCCIDENTAL Y CENTRAL & 27,1 & 23,1 & 31,2 & 18,8 & 8,1 & 10,7 & 39,7 & 37,0 & 42,4 & 12,5 & 5,9 & 6,6 \\
\hline MUNDO & 8,9 & 8,1 & 9,7 & 57,8 & 27,3 & 30,5 & 16,8 & 16,2 & 17,5 & 62,9 & 31,3 & 31,6 \\
\hline
\end{tabular}

Notas: Los datos se refieren a la clasificación por regiones que utiliza UNICEF. La categoría que engloba Europa Occidental, América del Norte y Australasia no es una región oficial de UNICEF, pero se utiliza para agrupar a todos los países que no pertenecen a otras regiones de UNICEF; se refiere a países de ingresos altos y medos altos localizados en Australasia, Europa y América del Norte en los cuales no actúa UNICEF.

Fuente: Instituto de Estadística de la UNESCO, agosto de 2014

Las causas de la inasistencia escolar son variadas, complejas y a menudo interrelacionadas, pero en este informe encuentra que hay un denominador común: los sistemas educativos y los entornos en los cuales están inmersos contribuyen con frecuencia a que algunos niños estén en desventaja. Por consiguiente, los progresos no han sido equitativos; de hecho, los niños y las niñas menos favorecidos siguen marginados, pese a los esfuerzos que se han realizado para lograr la educación para todos.

\section{LOS DESAFÍOS}

Los tambaleantes progresos mundiales hacia la educación primaria universal han puesto en evidencia diferencias significativas entre los países. Algunos todavía tienen que recorrer un largo camino antes de acercarse a la matriculación universal en la enseñanza primaria y a la conclusión (ver el Gráfico E2, basado en el Atlas electrónico de la UNESCO sobre los niños fuera de la escuela, disponible en http://on.unesco.org/oosc-mapES). El 50\% de todos los niños fuera de la escuela a nivel mundial pertenecen a un pequeño número de países, muchos de los cuales se caracterizan por la inestabilidad, los conflictos y la pobreza extrema. Sin embargo, pese a necesitar proporcionalmente más ayuda, estos países enfrentan grandes dificultades para acceder a los servicios de expertos externos y a apoyo financiero. La ayuda externa que han recibido países como Nigeria y la República Democrática del Congo, donde viven alrededor de 11 millones de niños en edad de asistir a la escuela primaria pero que no están escolarizados, ha sido desproporcionadamente baja.

Una situación similar se observa a nivel regional, donde un número relativamente pequeño de países tiene un porcentaje desproporcionadamente alto de niños fuera de la escuela (ver el Gráfico E3). En Oriente Medio y África del Norte, tres cuartas partes de los niños no escolarizados se encuentran en dos países, Sudán y Yemen. En Burkina Faso, Chad, Ghana, Malí, Níger y Nigeria, países de África Occidental y Central, la proporción es la misma. Datos de encuestas de hogares indican que aproximadamente la mitad de los niños fuera de la escuela de África Oriental y Meridional viven en Etiopía, Kenya, la República Unida de Tanzanía y Somalia. A su vez, en Asia Meridional, más del 50\% de los niños fuera de la escuela viven en Pakistán.

En otros países ha habido progresos tan asombrosos en materia de acceso a la educación que ya se encuentran en la "recta final" hacia la educación primaria universal. Pero precisamente en estos países los niños más desfavorecidos - "el último $10 \%$ ", como se les conoce - no se han beneficiado de esos resultados positivos. Estos países enfrentan un conjunto diferente de desafíos; de esta manera, 
Gráfico E2. Porcentaje de niños en edad de asistir a la educación primaria que se encuentra fuera de la escuela
Menos del 10\%
$10 \%-19,9 \%$
$20 \%-29,9 \%$
$30 \%$ o más
No hay datos

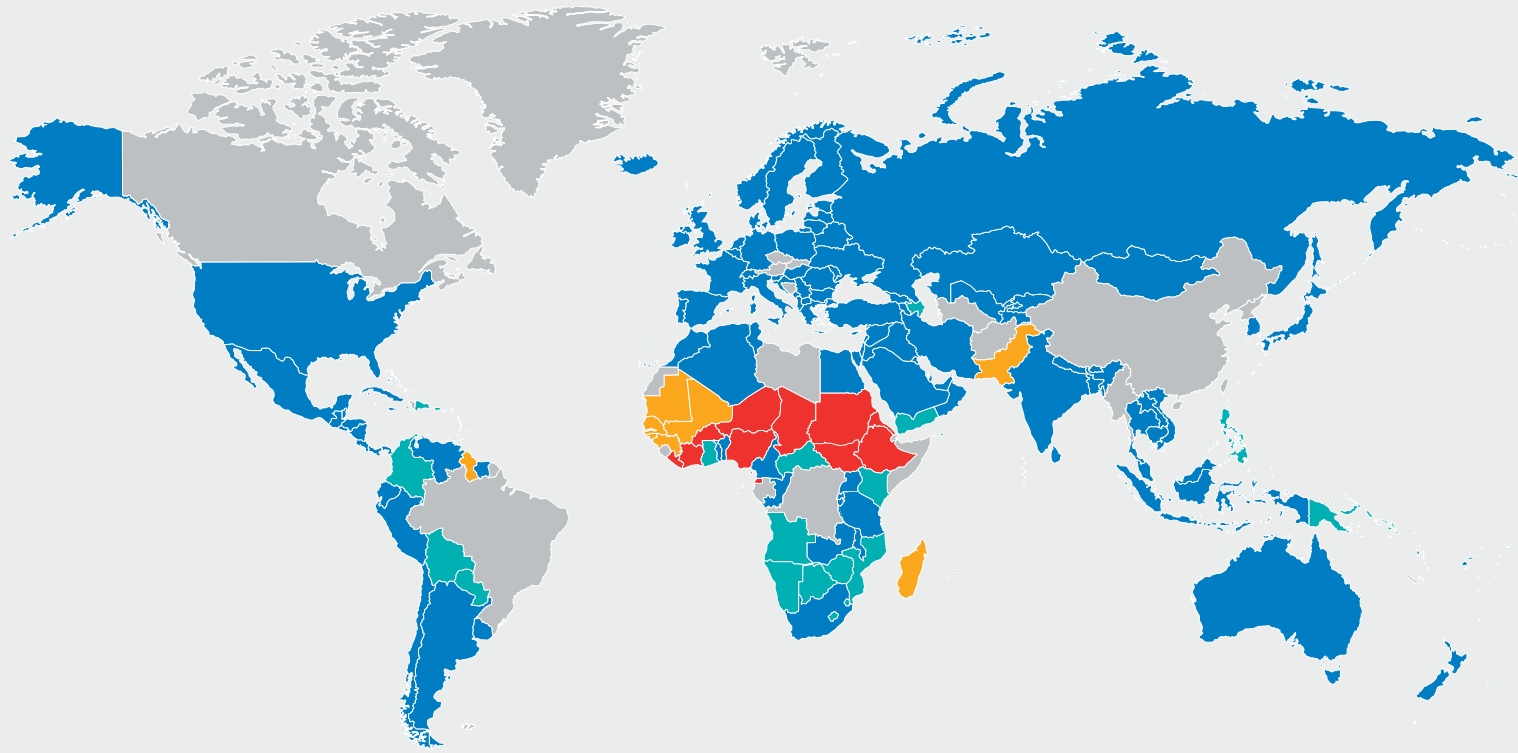

Fuente: Instituto de Estadistica de la UNESCO (http://on.unesco.org/oosc-mapES)

Gráfico E3. Países seleccionados con más de 0,5 millones de niños fuera de la escuela en edad de asistir a la educación primaria, por regiones, 2012 o el último año con datos disponibles

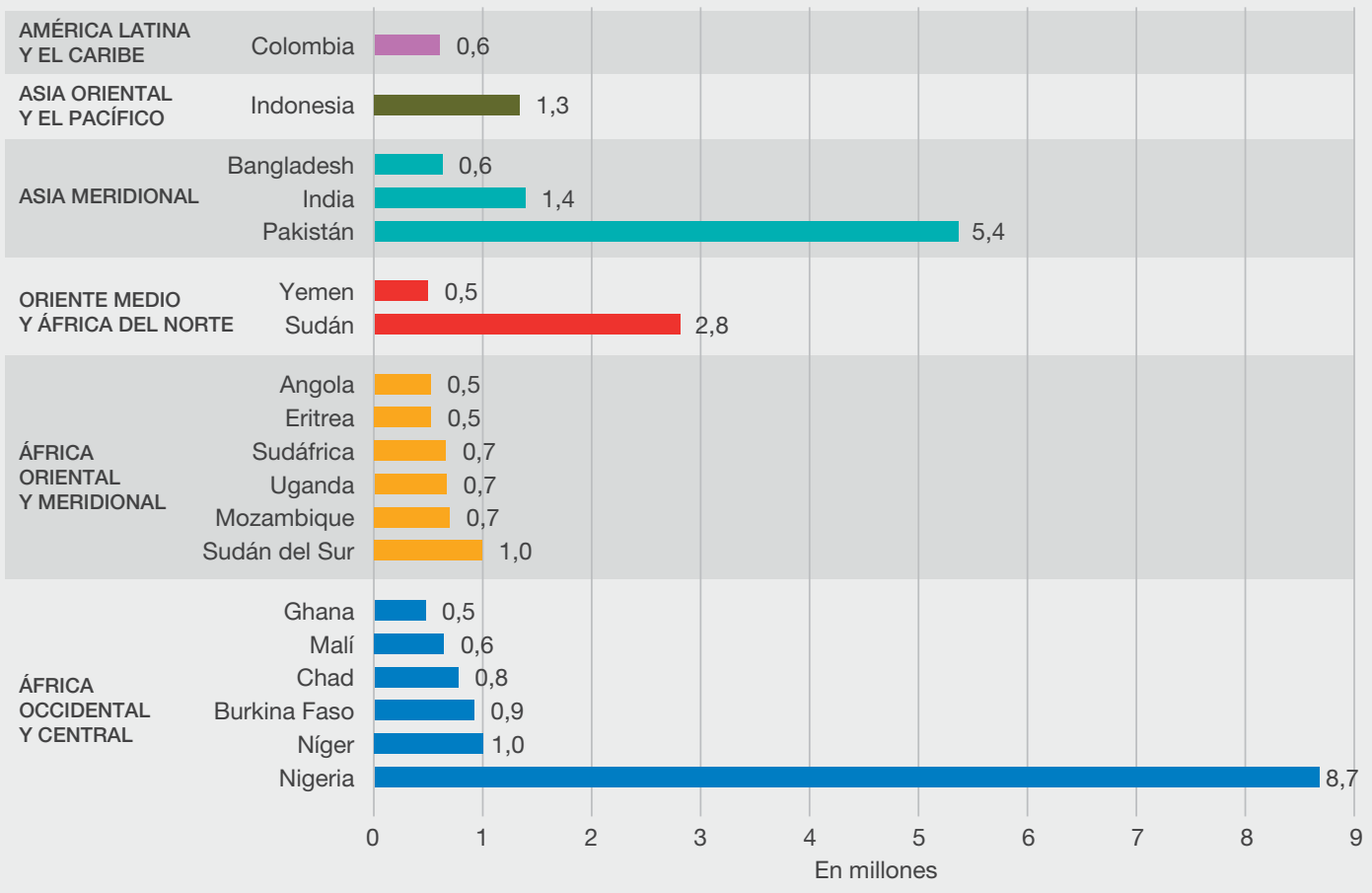

Notas: Los datos de Ghana corresponden a 2013; los datos de Angola, Chad, India, Sudán, Sudán del Sur y Uganda, a 2011; y los datos de Bangladesh y Nigeria, a 2010.

Fuente: Instituto de Estadística de la UNESCO, agosto de 2014 DataLink: http://dx.doi.org/10.15220/2014/ed/sd/7/f2.4 
podrían estar prestando una creciente atención a la educación preescolar y secundaria antes de concluir la labor relacionada con la enseñanza primaria para asegurar así un derecho fundamental para todos los niños.

Los niños fuera de la escuela que son más difíciles de alcanzar requieren medidas de política más complejas y, a menudo, más costosas. Aun cuando puedan representar solo un pequeño porcentaje de toda la población infantil, su número puede ser todavía sumamente alto. En el Brasil, por ejemplo, una tasa de niños fuera de la escuela de apenas un 2,4\% en 2009 significa que más de 730.000 niños en edad de asistir a la escuela primaria y al primer ciclo de la enseñanza secundaria siguen sin estar escolarizados (UNICEF e Instituto de Estadística de la UNESCO, 2012).

En el informe se subraya no solo la importancia de identificar y ubicar a los niños fuera de la escuela, sino también si han asistido alguna vez a la escuela y si es probable que lo hagan en el futuro (ver e/ Gráfico E4). El apoyo y el tipo de programas educativos que se requieren en países como Nigeria y Pakistán - donde la mayoría de los niños no escolarizados nunca accederán a la escuela - son muy distintos de los que se necesitan en países como India o Indonesia, donde la mayoría de estos niños ingresaron a la escuela pero la abandonaron antes de concluir los estudios. En otros países como Bolivia, Camboya y Etiopía, la mayoría de los niños que actualmente no están en la escuela empezarán a estudiar tarde, pero correrán un riesgo mayor de abandonar la escuela debido a que serán estudiantes con sobreedad.

Una mirada más profunda a los datos también revela la medida en que las desigualdades en aspectos como, por ejemplo, el nivel de ingresos familiares o el lugar de residencia, pueden incidir en una mayor marginación de los niños fuera de la escuela. En muchos países, estas tasas son significativamente más altas entre los niños de familias pobres del

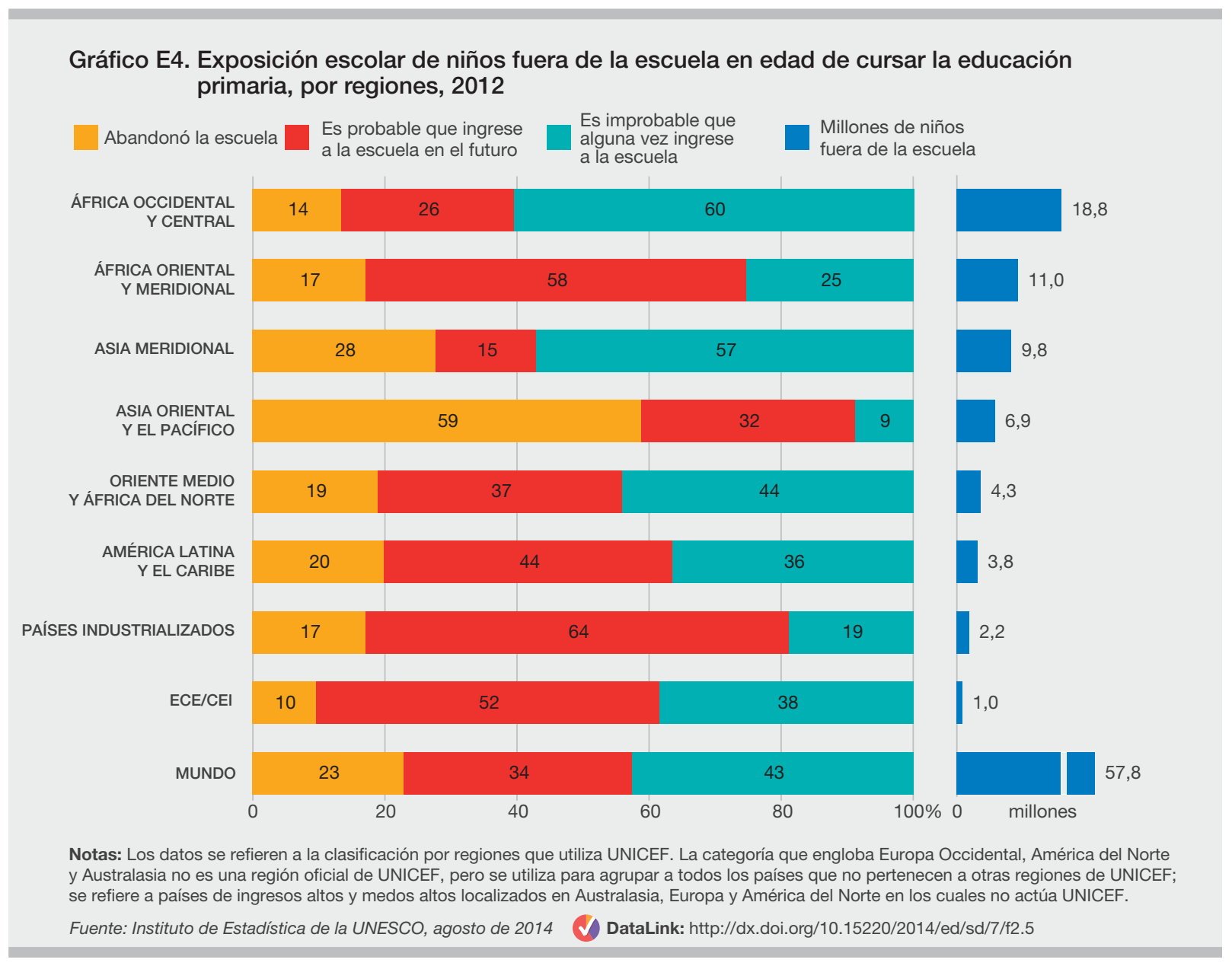


medio rural que entre los niños de familias más ricas del medio urbano. Pero la verdadera magnitud de esta disparidad se aprecia aún más al tomar en consideración los datos sobre la exposición a la escuela de estos diferentes grupos. Es previsible que los niños de familias urbanas ricas que actualmente no asisten a la escuela comenzarán a hacerlo en algún momento, incluso si la abandonan prematuramente. En cambio, los niños pobres que viven en las zonas rurales suelen tener muy pocas probabilidades de ingresar alguna vez en su vida a un aula escolar. En Nigeria, por ejemplo, dos tercios de los niños en edad de cursar la enseñanza primaria, y pertenecientes a las familias más pobres, no están escolarizados. De ellos, es probable que cerca del $90 \%$ nunca llegue a matricularse en la escuela (UNICEF e Instituto de Estadística de la
UNESCO, 2012f). Por el contrario, solo el 5\% de los niños más ricos se encontraban fuera de la escuela y se prevé que la mayoría ingresen a una en el futuro. Para dar una idea de la magnitud de estas disparidades, este informe viene acompañado de una herramienta interactiva de exploración de datos en línea que presenta la información más reciente de los países que participan en la Iniciativa Global por los Niños fuera de la Escuela (ver http://on. unesco. org/oosci-globalES).

\section{El informe Fixing the Broken Promise of Education} for All va más allá de las cifras para explorar los obstáculos que privan a los niños de la oportunidad de educarse. En primer lugar, pone de relieve la situación de los niños en los países afectados por conflictos, que representan solo el $20 \%$ de los

\section{Gráfico E5. Porcentaje de niños de 7 a 14 años fuera de la escuela, por situación laboral del niño, en determinados países}

Porcentaje de niños de 7 a 14 años sin escolarizar, por situación laboral del niño, en determinados países

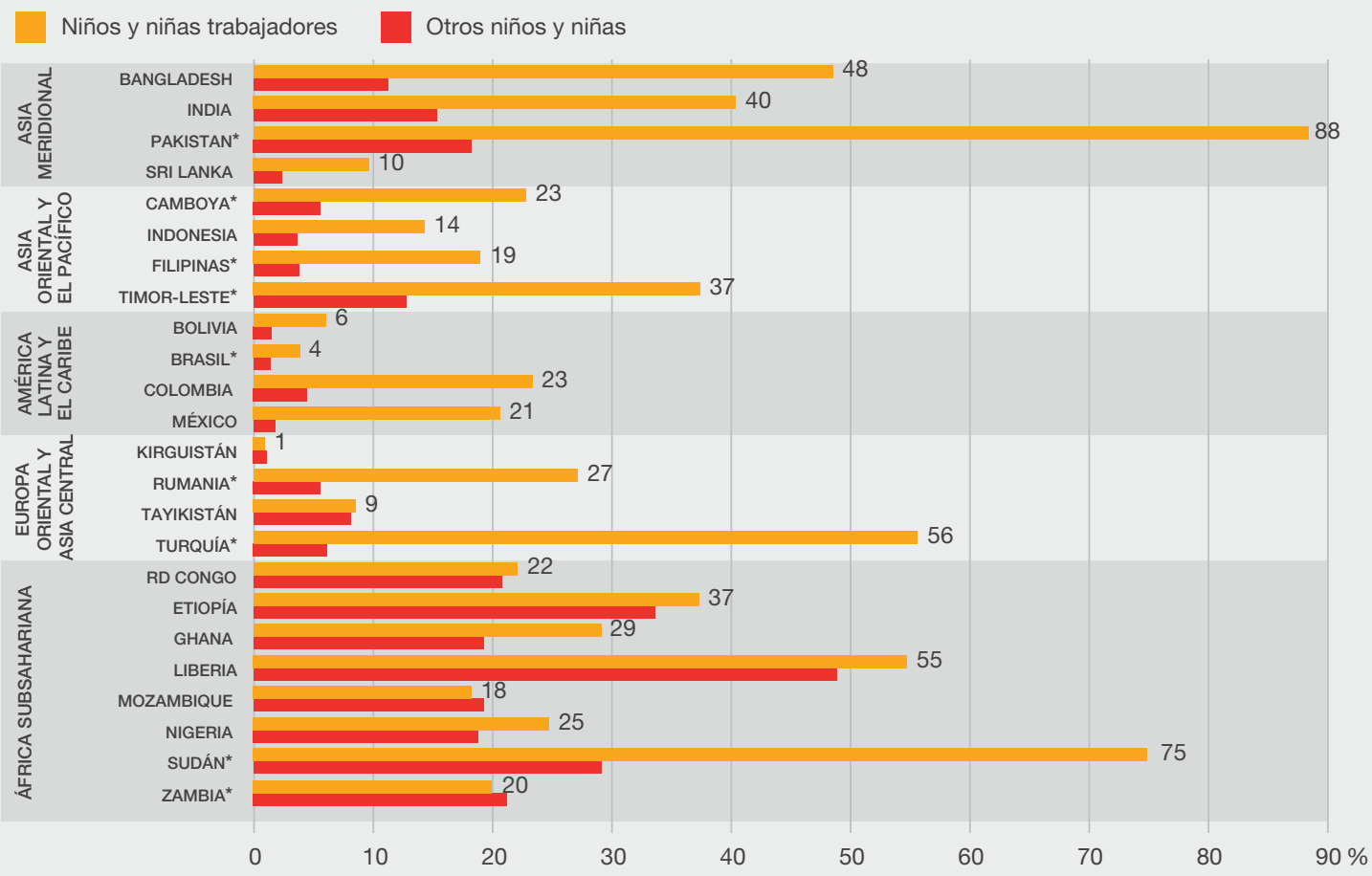

Notas: * Indica las estadísticas sobre el trabajo infantil que difieren de la definición internacional del trabajo infantil debido a las limitaciones de la encuesta en los hogares o el censo utilizado. Los datos para Timor-Leste, Pakistán y Sudán abarcan niños de 10 a 14 años, y para Turquía de 6 a 14 años. Los datos de Camboya, Filipinas, Pakistán, Rumania y Zambia no incluyen información acerca de las horas dedicadas a las tareas del hogar: la definición del trabajo infantil en estos países se basa sólo en las horas pasadas en el empleo. Los datos de Sudán no incluyen información sobre las horas pasadas en el empleo y en las tareas del hogar; la definición del trabajo infantil se basa sólo en las horas pasadas en el empleo. Los datos para Brasil utilizan la definición internacional, aunque la legislación nacional brasileña no permite el trabajo ligero para los niños de 12 a 14 años. Fuentes: Understanding Children's Work calculations based on Bangladesh Labour Force Survey, 2005-2006; Bolivia Encuesta de Trabajo Infantil (SIMPOC), 2008; Brazil Pesquisa Nacional por Amostra de Domicílios, 2011; Cambodia Labour Force and Child Labour Survey (SIMPOC), 2012; Colombia GEIH: Módulo de Trabajo Infantil, 2012; DR Congo MICS, 2010; Ethiopia DHS, 2011; Ghana MICS, 2006; India DHS, 2005-2006; Indonesia Child Labour Survey (SIMPOC), 2009; Kyrgyzstan Child Labour Survey, 2007; Liberia DHS, 2007; Mexico Encuesta Nacional de Ocupación y Empleo: Módulo de Trabajo Infantil, 2011; Mozambique MICS, 2008; Nigeria MICS, 2011; Pakistan Labour Force Survey 2007-2008; Philippines Labour Force Survey (SIMPOC), 2001; Romania Child Labour Curve (SIMPOC), 2000; Sri Lanka Child Activity Survey (SIMPOC), 1999; Sudan Fifth Population and Housing Census, 2008; Tajikistan MICS, 2005; Timor-Leste Survey of Living Standards, 2007; Turkey Child Labor Survey (SIMPOC), 2006; Zambia Labour Force Survey, 2008 DataLink: http://dx.doi.org/10.15220/2014/ed/sd/7/f3.5 


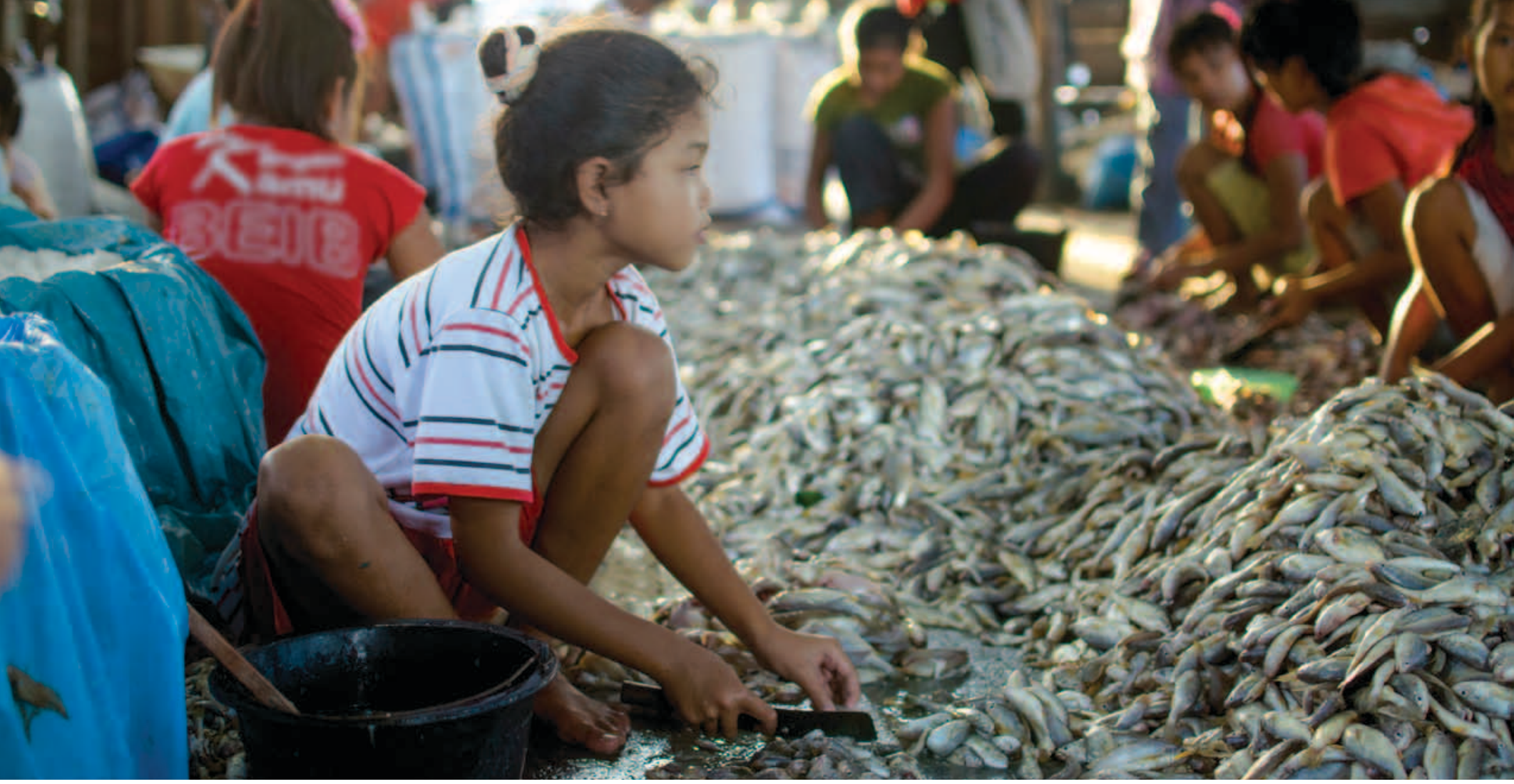

\section{Recuadro E1. Explorar los datos}

Los niños a los que es más difícil atender aun siguen fuera de la escuela. Son pobres, viven en las zonas rurales y usualmente son niñas. No obstante, la situación es diferente en cada país. El explorador interactivo de datos del Instituto de Estadística de la UNESCO ilustra la multiplicidad y superposición de las barreras a la educación en los 27 países que participaron en la Iniciativa Global por los Ninos fuera de la Escuela. Para conocer más a fondo las razones de la injusta exclusión de estos niños invisibles y carentes de voz, sírvase visitar http://on.unesco.org/oosci-globalES

El Atlas electrónico de la UNESCO sobre los niños afuera de la escuela ofrece información adicional: http://on.unesco.org/oosc-mapES

niños en edad de cursar la primaria a nivel mundial, pero el $50 \%$ de los niños fuera de la escuela en todo el mundo. En segundo lugar, explica que los arraigados roles asignados a cada género con frecuencia determinan si el niño o la niña se matricula - y permanece - en la escuela. En tercer lugar, la necesidad que tienen las familias de que los niños trabajen compite con la aspiración de que se eduquen (ver el Gráfico E5). En cuarto lugar, demasiados niños quedan marginados de la educación debido a que las clases se imparten en idiomas que no hablan o no comprenden. Por último, los niños con discapacidades afrontan una multitud de obstáculos - desde la ausencia de una educación inclusiva hasta aulas totalmente inaccesibles - que les impiden gozar de su derecho a la educación. Los problemas son resultado de la combinación de factores que retrasan o restringen el acceso a la educación, o que hacen imposible la escolarización.

El informe analiza la disponibilidad y la calidad de los datos de los países participantes en la Iniciativa Global por los Niños fuera de la Escuela, y muestra que la capacidad para responder los complejos obstáculos a la educación universal se ve limitada por una seria insuficiencia de datos. Según estudios realizados por los países, faltaba información crucial sobre los niños más marginados, o la información era de baja calidad, reafirmando la necesidad de mejorar la recopilación y la selección de los datos, así como también la manera en que se utilizan para orientar las políticas. En las circunstancias actuales, la naturaleza intersectorial de los problemas y las medidas relacionadas con los niños fuera de la escuela implica que la producción de los datos, la presentación de informes y, por consiguiente, la formulación de políticas para este grupo, a menudo carecen del compromiso sostenido y coordinado que se requiere para beneficiar a todos los niños. 


\section{SUBSANAR LA PROMESA INCUMPLIDA}

"Hay muchas razones [que explican por qué hay niños afuera de la escuela]. Ante todo, razones económicas. La mayoría de los padres congoleños son pobres e incluso los que trabajan ganan poco y el empleador decide cuándo entregarles el salario. Es difícil arreglárselas; con el salario debes pagar el alquiler, la educación, los gastos médicos, el transporte y todo lo demás. Los padres dicen: no puedo pagar todo y debo elegir a uno o dos de mis hijos [para asistir a la escuela]... los otros deben esperar".

Educador, República Democrática del Congo

(UNICEF e UIS, 2013b)

Para subsanar la promesa incumplida, los países cuyas poblaciones de niños fuera de la escuela siguen siendo altas deben buscar un equilibrio entre las intervenciones destinadas específicamente a los niños más marginados y las reformas más amplias en todo el sistema educativo. Por ejemplo, en Eritrea, Nigeria y Sudán del Sur, las políticas focalizadas no pueden compensar, por sí solas, la debilidad de sus sistemas educativos. El hincapié debe recaer en las inversiones orientadas a fortalecer y expandir estos sistemas, a garantizar que haya suficientes escuelas sin costos directos o indirectos para los alumnos, y a prestar especial atención a la inclusión y la calidad de la enseñanza

Sin embargo, los países que se encuentran en la recta final tienen la urgente necesidad de tomar medidas enfocadas especialmente en la superación de los obstáculos que mantienen fuera del sistema escolar a los niños más inaccesibles. No será posible beneficiar a los niños de países como Brasil, Sri Lanka y Turquía por medio de estrategias que sean "más de lo mismo", ampliando aún más los sistemas educativos existentes. Más bien, debe buscarse una mayor equidad en la educación, pasando de los sistemas que asignanlos recursos uniformemente a uno que permita asignarlos de acuerdo con las necesidades. Este cambio es esencial para garantizar que todos los niños tengan la posibilidad de educarse en condiciones de igualdad.

En todo el mundo, la pobreza familiar se asocia con los obstáculos más persistentes para la educación. Los altos costos directos e indirectos de
La mayoría de los países requieren tres prioridades de política: hacer amplias inversiones para fortalecer y ampliar los sistemas educativos, prestar especial atención a la inclusión y la calidad de la educación que ofrecen, y poner en marcha intervenciones focalizadas en los niños que están en situaciones más difíciles de alcanzar la escolaridad impiden a los niños realizar su derecho a la educación. En el informe se reseñan diversas intervenciones indispensables para todo el sistema, pues tienen por objeto mejorar la disponibilidad, asequibilidad económica y calidad de la educación para todos los niños. Asimismo, se explica que la pobreza actúa de múltiples maneras, intensificando otras fuentes de desventajas. La doble desventaja de la pobreza ocupa un lugar prominente en las medidas que se exponen en el informe para superar cinco barreras a la educación para todos.

Simenda tuvo que esforzarse al máximo para sacar adelante sus estudios en la escuela secundaria a la que asistía, en una zona rural de Namibia, hasta que no le diagnosticaron un impedimento auditivo. Sus maestros aprendieron estrategias para ayudarle en sus estudios, como permitirle sentarse donde pudiera oír y verificar que estuviera siguiendo las clases. Su rendimiento escolar mejoró tanto después de dos semestres que llegó a ocupar el octavo puesto entre un grupo de 30 estudiantes.

(UNICEF e Instituto de Estadística de la UNESCO, 2014)

- Los conflictos. Es necesario un enfoque de tres componentes para que una educación de buena calidad llegue a formar parte de reformas sociales más amplias tendientes a prevenir los conflictos, posibilitar la continuación de la enseñanza durante los conflictos y garantizar que las reformas educativas posteriores a los conflictos propicien una recuperación económica y social que pueda evitar el resurgimiento de la violencia. 
- La discriminación por razón del género. La prioridad es conseguir que incluso la niña más vulnerable y desfavorecida tenga acceso a una escuela que no solo esté ubicada cerca de su hogar, sino que satisfaga sus necesidades básicas en materia de seguridad, privacidad e higiene, que ofrezca la mejor educación posible, y que valore su presencia y sus potencialidades.

- El trabajo infantil. Con el propósito de incrementar la asistencia a la escuela, el trabajo infantil debe reducirse. Teniendo en cuenta los fuertes nexos entre la pobreza y el trabajo infantil, eliminar los costos directos e indirectos asociados con la educación es la manera más efectiva de lograrlo. Hacen falta sistemas educativos más flexibles y sensibles a las necesidades de los niños, al igual que mejores entornos de aprendizaje, para atraer a la escuela a los niños que trabajan y lograr que no abandonen sus estudios.

- Los problemas relacionados con los idiomas. Los programas educativos en las lenguas vernáculas de los alumnos tienen mayores niveles de participación, éxito y disfrute - así como también de involucramiento de los progenitores, y menores niveles de repetición y abandono, sobre todo entre las niñas. Las escuelas que imparten la enseñanza en idiomas que los niños entienden permiten a los alumnos participar y demostrar lo que han aprendido, a la vez que favorecen la participación de sus familias.

- Los obstáculos sociales, institucionales y ambientales relacionados con las discapacidades. La educación de los niños con discapacidad depende de la eliminación de las numerosas barreras que se oponen a su educación: desde la falta de acceso físico a las aulas hasta el estigma que los mantiene ocultos en sus hogares. También requiere prestarles el apoyo adecuado y comprender sus fortalezas, limitaciones y necesidades, con fundamentación en datos sólidos y comparables.

Para ponerlo en palabras simples, el optimismo más desbordante no hará que los 121 millones de niños, niñas y adolescentes que hay en el mundo y

\section{Recuadro E2. Remediar la insuficiencia de datos}

Llegar a los niños que se encuentran fuera de la escuela exige tener una idea clara de quiénes son, dónde se encuentran y por qué no están en las salas de clase. Una base de información de gran amplitud constituye un fundamento sólido para el diseño de políticas efectivas que se traduzcan en un aumento de la matriculación escolar y en apoyo a los niños para que terminen con éxito un ciclo completo de enseñanza básica de alta calidad.

Una manera práctica de identificar a los niños que están fuera de la escuela es hacer un mejor uso de las fuentes de datos que ya existen. Un importante hallazgo de la Iniciativa Mundial por los Niños fuera de la Escuela es que las encuestas de hogares son un recurso insuficientemente utilizado, que proporciona información sobre los perfiles de estos niños por género, lugar de residencia, ingresos familiares, educación de los progenitores y otras características personales y familiares. No obstante, los ministerios de educación de los países raramente utilizan estas encuestas. Una mayor colaboración intersectorial mejoraría el acceso y el análisis de esta valiosa fuente de información para la formulación de políticas educativas. Para tener la seguridad de que las preguntas y los datos sobre educación que se obtienen en estas encuestas son correctos y útiles, durante la elaboración y el análisis de las encuestas de hogares convendría consultar con funcionarios de los ministerios de educación.

Los datos administrativos, con su cobertura relativamente completa de todos los alumnos en todas las escuelas, tienen grandes posibilidades, por ejemplo, de observar con mayor detalle zonas con altas tasas de estudiantes con sobreedad o que dejan la escuela prematuramente, antes de concluir la educación básica. Con las recientes mejoras en los sistemas de información para la gestión de datos sobre educación, los datos administrativos pueden emplearse para identificar a los niños fuera de la escuela.

Uno de los principales resultados de la Iniciativa Mundial por los Niños fuera de la Escuela ha sido el mayor uso de ambas fuentes de datos para el análisis de la situación de estos niños. Lo anterior ha propiciado la reflexión y la acción en los planos nacional e internacional, con vistas a mejorar la cobertura, la precisión y la utilización de los datos sobre los niños que no asisten a la escuela. 
se encuentran fuera de la escuela puedan acceder a la educación mientras que no se adopten políticas para abordar estos desafíos que se enfrentan.

Ya sea que se trate de expandir los sistemas educativos, de concentrarse en los niños más marginados o de combinar estas dos medidas, los encargados de formular políticas tendrán que encontrar una enorme cantidad de recursos adicionales. El informe Fixing the Broken Promise of Education for All (Subsanar la promesa incumplida de la Educación para Todos) no suministra una cifra global en dólares para los recursos que permitirían ofrecer una educación primaria universal. Más bien, se ciñe al enfoque de la Iniciativa Mundial por los Ninos fuera de la Escuela, que otorga especial importancia al análisis adaptado al contexto local. En el informe se describen dos estrategias innovadoras de determinación de costos para facilitar a los países la toma de decisiones sobre los niños que no asisten a la escuela. En la República Democrática del Congo, análisis innovadores de costos centrados en la equidad ofrecen una visión realista de la escala de los recursos que se precisan para que todos los niños fuera de la escuela en este país accedan a la enseñanza: 111 millones de dólares anuales, lo que equivale a una cuarta parte del gasto público total del país en educación en 2011. Además de instar a incrementar los gastos generales, el informe hace un llamado para invertir más en intervenciones de eficacia comprobada. Sostiene que el esfuerzo vale la pena, habida cuenta de los beneficios a largo plazo para el bienestar económico y social de todos los países.

El informe Fixing the Broken Promise of Education for All confirma la necesidad urgente de reavivar el interés en los niños fuera de la escuela. Y lo hace, precisamente, en momentos en que la comunidad internacional está definiendo nuevos objetivos y metas, con el argumento de que los niños que no están asistiendo a la escuela deben ocupar un lugar central en el nuevo marco mundial para el desarrollo. 


\section{Referencias}

Instituto de Estadística de la UNESCO e Informe de Seguimiento de la Educación para Todos en el Mundo (2014). "Progress in getting all children to school stalls, but some countries show the way forward". Documento de política 14 del Informe de Seguimiento, ficha descriptiva 28 del Instituto de Estadística de la UNESCO. París: UNESCO.

UNICEF e Instituto de Estadística de la UNESCO (2014). Eastern and Southern Africa Regional Report (Informe Regional de África Oriental y Meridional). Iniciativa Mundial sobre los Niños Sin Escolarizar. Nairobi: UNICEF e Instituto de Estadística de la UNESCO. Disponible en: http://dx.doi.org/10.15220/uis-unicef-reg-esar-2014-en

UNICEF e Instituto de Estadística de la UNESCO (2013a). Out-of-School Children in Sri Lanka (Niños desescolarizados en Sri Lanka). Iniciativa Mundial sobre los Niños Sin Escolarizar. Colombo: UNICEF e Instituto de Estadística de la UNESCO. Disponible en: http://dx.doi.org/10.15220/uis-unicef-cntry-sri-lanka-2013-en

UNICEF e Instituto de Estadística de la UNESCO (2013b). Rapport de l'enquête nationale sur les hors de l'école [República Democrática del Congo]. Iniciativa Mundial sobre los Niños Sin Escolarizar. Kinshasa: UNICEF e Instituto de Estadística de la UNESCO. Disponible en: http://dx.doi.org/10.15220/uis-unicef-cntry-congo-2013-fr

UNICEF e Instituto de Estadística de la UNESCO (2012). Completar la escuela: Un derecho para crecer, un deber para compartir. América Latina y el Caribe. Iniciativa Mundial sobre los Niños Sin Escolarizar. Panamá: UNICEF e Instituto de Estadística de la UNESCO. Disponible en: http://dx.doi.org/10.15220/978-92-806-4653-5-sp 
TODOS EN LA ESCUELA

\section{Iniciativa mundial sobre niños y niñas que no asisten a la escuela}

UNICEF Y INSTITUTO DE ESTADÍSTICA DE LA UNESCO

Subsanar la Promesa Incumplida de la Educación para Todos, un informe producido por el Instituto de Estadística de la UNESCO y UNICEF, presenta las últimas pruebas estadísticas procedentes de los registros administrativos y las encuestas en los hogares que permiten identificar mejor a los niños sin escolarizar y las razones que explican su exclusión de la educación. Trata de dotar de contenido a las políticas necesarias para llegar a estos niños y cumplir finalmente con la promesa de la educación para todos.

Sobre la base de una serie de estudios nacionales y regionales y análisis de políticas realizados por importantes expertos, el informe explica por qué disponer de mejores datos y colaborar de forma intersectorial son dos cuestiones fundamentales para el diseño de intervenciones eficaces que superen los obstáculos a los que hacer frente los niños y los adolescentes sin escolarizar. Al mismo tiempo que destaca la senda futura de las políticas a nivel de sistema para mejorar la calidad de la educación y lograr que sea asequible, el informe también presenta la información necesaria para llevar a cabo enfoques selectivos que faciliten abordar los efectos multiplicadores de las desventajas que sufren los niños atrapados en conflictos armados, las niñas, los niños y niñas que trabajan, los niños y niñas con discapacidad, o los miembros de las minorías étnicas o lingüísticas

Este informe presenta una hoja de ruta para mejorar los datos, las investigaciones y las políticas necesarias para catalizar la acción en favor de los niños sin escolarizar, en un momento en que el mundo inicia un nuevo programa de desarrollo para la educación.

ISBN 978-92-9189-165-8 